\title{
Introduction to the second issue of Social Network Analysis and Mining journal: scientific computing for social network analysis and dynamicity
}

\author{
Reda Alhajj • Nasrullah Memon
}

Published online: 29 March 2011

(C) Springer-Verlag 2011

Welcome to the second issue of the Social Network Analysis and Mining journal. In this issue, we will continue to demonstrate the high standard of the SNAM journal which started by publishing five well written papers in the first issue. We are delighted to witness the journal gaining popularity since the first articles were published online. The number of visitors to the online version of the journal is increasing rapidly and the number of downloaded articles shows the interest in the journal. We much appreciate the increased interest in the journal. We are receiving more submissions than expected. Fortunately, we are maintaining the short turnaround time for the reviewing process; humble thank you to the expert reviewers and board members who dedicate their valuable time and effort to submit timely detailed and constructive reports.

Readers of this issue will enjoy the research achievements reported mostly by computer scientists who are experts in social network analysis and mining. This shows how the research in social networks has attracted considerable attention outside the basic disciplines where it originated, i.e., sociology and anthropology. We witness the application of the social network methodology in variety of disciplines. Market basket analysis is one good

R. Alhajj ( $\varangle)$

Department of Computer Science, University of Calgary,

Calgary AB T2N 1N4, Canada

e-mail: alhajj@ucalgary.ca; rsalhajj@gmail.com

R. Alhajj

Department of Computer Science, Global University,

Beirut, Lebanon

\section{N. Memon}

The Maersk Mc-Kinney Moller Institute, University of Southern Denmark, Campusvej 55, 5230 Odense M, Denmark

e-mail: memon@mmmi.sdu.dk application covered in this issue. It is even hard to think of a discipline which cannot benefit from the richness of the social network methodology.

The social network methodology has been well received in the scientific community due to several reasons, including the strong foundations in science and the tremendous development in the information technology. Basic sciences influenced the social network methodology from data collection, to network construction, to network dynamics, to network analysis, to results interpretation, etc. Good background in graph theory, linear algebra and statistics is necessary for successful application of the social network methodology. On the other hand, the development in information technology allowed researchers to consider scalability of the analyzed social networks. Automating the analysis and the sophisticated computing infrastructure and methods have direct influence on the successful research in network dynamics at large. Further, the development of Web 2.0, web services and web semantics has produced the current popularity of the terms "online social networks" and "social media". People are heavily involved in online social networks without knowing how they are structured or evolve. The first paper in this issue describes a novel method for analyzing online friendship networks.

We decided to have the SNAM journal open to both technical and non-technical articles in order to cover the multidisciplinary nature of the research and hence help in meeting the expectations of the wide spectrum of readers, including both researchers and practitioners. Though the papers in this issue are mainly written by computer scientists, we have already accepted some other less technical articles, some of which have appeared in the first issue and others will be included in future issues.

The current issue contains five well written papers covering various aspects of social networks analysis and 
mining. Some are revised and extended versions of best papers from the International conference on Advances in Social Network Analysis and Mining 2009 (ASONAM 2009) which was held in Athens, Greece in July 2009. We would like to thank all the contributing authors for the wonderful papers that will turn this second issue into a valuable source for researchers and practitioners.

The first paper: "Spectral Counting of Triangles via Element-Wise Sparsification and Triangle-Based Link Recommendation" by Charalampos E. Tsourakakis, Petros Drineas, Eirinaios Michelakis, Ioannis Koutis, and Christos Faloutsos, studies the problem of counting triangles in large, power-law networks and its applications to online social networks. Contributions described in the paper include: (1) a simple method for making link recommendations in online social networks based on the number of triangles, and (2) a procedure for estimating triangles using sketches.

The second paper: "Communities and Hierarchical Structures in Dynamic Social Networks: Analysis and Visualization" by Frederic Gilbert, Paolo Simonetto, Faraz Zaidi, Fabien Jourdan and Romain Bourqui, presents a system to analyze dynamic social networks. The proposed system is based on dynamic graph discretization and graph clustering. It detects major structural changes taking place in social communities over time and reveals hierarchies by highlighting influential actors in a social network.

The third paper: "Market Basket Analysis with Networks" by Troy Raeder and Nitesh V. Chawla, models the data as a product network and discovers expressive communities (clusters) in the data, which can then be targeted for further analysis. The authors developed an interestingness measure for analyzing communities of products; they show that the new measure isolates useful, actionable communities. Finally, the authors build upon their experience with product networks to propose a comprehensive analysis strategy by combining both traditional and network-based techniques.

The fourth paper: "Understanding Actor Loyalty to Event-Based Groups in Affiliation Networks" by Hossam Sharara, Lisa Singh, Lise Getoor, and Janet Mann, introduces a method for analyzing the temporal dynamics of affiliation networks. The authors define affiliation groups which describe temporally related subsets of actors. They describe an approach for exploring changing memberships in the affiliation groups over time. To model the dynamic behavior in the affiliation networks, the authors consider the concept of loyalty and introduce a measure that captures an actor's loyalty to an affiliation group as the degree of 'commitment' the actor shows to the group over time.

The fifth paper: "Seeing similarity in the face of difference: enabling comparison of online production systems" by Claudia Muller-Birn, Benedikt Meuthrath, Andreas Erber, Sebastian Burkhart, Anne Baumgraß, Janette Lehmann, and Robert Schmidl, presents an approach to model, analyze and visualize online production systems, such as Wikipedia, open source software development processes, and Flickr. The authors interpret online production systems as social information spaces, and describe them with a generic vocabulary that is implemented in software.

Last but not least, we would like to mention the hard workers behind the scene who have significant unseen contributions to the success. Various people from Springer deserve large credit for their help and support in all the issues related to the SNAM journal in general and in producing this second issue in particular. We would like to thank Stephen Soehnlen for his generous support in terms of time and effort required to keep the momentum. Unfortunately, Mhanilet de Leon is no longer associated with the SNAM journal. We extend warm thanks to her; she has been very patient in handling our requests and in following up with the submissions. She has been recently replaced by Inaoba Singh who will be the main contact with the authors in handling the submissions system; we welcome Inaoba. Finally, we would like to thank Philipp Kammerer from the production department for doing an amazing job taking care of the accepted papers, putting the issues together and alerting us whenever necessary.

Reda Alhajj and Nasrullah Memon

Editors-in-Chief

March 2011 